\title{
Axial motion and scalar transport in stretched spiral vortices
}

\author{
D. I. Pullin ${ }^{a)}$ \\ Graduate Aeronautical Laboratories 105-50, California Institute of Technology, Pasadena, California 91125
}

T. S. Lundgren

Department of Aerospace Engineering and Mechanics, University of Minnesota, Minneapolis, Minnesota 55455

(Received 29 November 2000; accepted 10 May 2001)

\begin{abstract}
We consider the dynamics of axial velocity and of scalar transport in the stretched-spiral vortex model of turbulent fine scales. A large-time asymptotic solution to the scalar advection-diffusion equation, with an azimuthal swirling velocity field provided by the stretched spiral vortex, is used together with appropriate stretching transformations to determine the evolution of both the axial velocity and a passive scalar. This allows calculation of the shell-integrated three-dimensional spectra of these quantities for the spiral-vortex flow. The dominant term in the velocity (energy) spectrum contributed by the axial velocity is found to be produced by the stirring of the initial distribution of axial velocity by the axisymmetric component of the azimuthal velocity. This gives a $k^{-7 / 3}$ spectrum at large wave numbers, compared to the $k^{-5 / 3}$ component for the azimuthal velocity itself. The spectrum of a passive scalar being mixed by the vortex velocity field is the sum of two power laws. The first is a $k^{-1}$ Batchelor spectrum for wave numbers up to the inverse Batchelor scale. This is produced by the axisymmetric component of the axial vorticity but is independent of the detailed radial velocity profile. The second is a $k^{-5 / 3}$ Obukov-Corrsin spectrum for wave numbers less than the inverse Kolmogorov scale. This is generated by the nonaxisymmetric axial vorticity and depends on initial correlations between this vorticity and the initial scalar field. The one-dimensional scalar spectrum for the composite model is in satisfactory agreement with experimental measurement. (C) 2001 American Institute of Physics.
\end{abstract}

[DOI: $10.1063 / 1.1388207]$

\section{INTRODUCTION}

Quantitative descriptions of turbulence based on ensembles of structured vortical solutions of the Navier-Stokes equations have achieved some success in modeling various aspects of turbulence fine scales. ${ }^{1-7}$ In these models, turbulence is envisioned as a collection of vortices, each one of which is being stretched along its axis by the collective velocity field of the vortex ensemble. Vortices generally have larger angular velocity near their centers and this difference in rotation rates causes nonuniformities in vorticity to be deformed into vortex layers which spiral around the vortex. This differential rotation, and the stretching also, causes a lateral contraction whereby the spacing between spiral vorticity layers decays in a way that produces a cascade of energy to smaller scales where it is dissipated by heat. By neglecting the curvature of the vortices and taking the stretching to be homogeneous, it is possible to find approximate solutions of the Navier-Stokes equations which describe this process. The corresponding structures may be thought of as a form of generalized and nonaxisymmetric Burgers vortices. This model has been used ${ }^{1}$ to derive the Kolmogorov $k^{-5 / 3}$ energy spectrum, and has been extended

\footnotetext{
a) Author to whom correspondence should be addressed; Electronic mail: dale@galcit.caltech.edu
}

and used ${ }^{3-8}$ to calculate vorticity and velocity-derivative moments, one-dimensional spectra and other small-scale properties of turbulence.

The stretched spiral-vortex model contains several severe simplifications. One of these is that the vorticity is assumed to be everywhere aligned with the vortex axis and with the principal extensional eigenvector of the stretching strain field. There is thus no rotational axial flow. This is inconsistent with recent observations of the vorticity on spiral vortex sheets in homogeneous turbulence. ${ }^{9}$ Solutions of the Navier-Stokes equations describing the evolution of a uniform shear flow with streamlines parallel to an embedded diffusing line vortex were obtained by Pearson and Abernathy, ${ }^{10}$ Moore, ${ }^{11}$ and Kawahara et al. ${ }^{12}$ This flow describes radially diffusing axial vorticity which drives the evolution of the axial velocity of the shear flow. Pearson and Abernathy ${ }^{10}$ noted a decoupling in the equations describing the axial vorticity and the axial velocity for flows of this type, where the initial conditions of all vorticity fields are independent of an axial coordinate. The idea was extended by Fokas et al. ${ }^{13}$ to a more general setting which included stretching of the axial vorticity. It is of interest to determine if the stretched-spiral vortex can accommodate nonaxial vorticity, and further, if this alters its spectral properties at large wave numbers. This has bearing on the utility of the stretched-spiral vortex as a viable model for the small scales of turbulence. 
In the sequel it will be seen that the evolution of the axial velocity for flows of the stretched-spiral vortex type is formally related to the mixing of a passive scalar, thus allowing a common treatment of both problems. There are several well established results for the scalar spectral density. For homogeneous isotropic turbulence the scalar spectrum in the inertial convective range is expected to be given by the Obukov-Corrsin form (see Tennekes and Lumley ${ }^{14}$ )

$$
E_{c}(k) \sim \epsilon_{c} \epsilon^{-1 / 3} k^{-5 / 3}, \quad L^{-1} \ll k \ll\left(\frac{\nu^{3}}{\epsilon}\right)^{-1 / 4},
$$

where $k$ is wave number, $\epsilon_{c}$ is the rate of dissipation of the scalar $c, \epsilon$ is the energy dissipation rate, $L$ is the integral scale and $\nu$ is the kinematic viscosity. This result has been established by dimensional analysis paralleling that used to obtain the Kolmogorov $k^{-5 / 3}$ energy (velocity) spectrum. When the scalar diffusivity $D \ll \nu$, Batchelor ${ }^{15}$ derived the spectrum

$$
E_{c}(k) \sim \epsilon_{c} \nu^{1 / 2} \epsilon^{-1 / 2} k^{-1}, \quad\left(\frac{\nu^{3}}{\epsilon}\right)^{-1 / 4} \ll k \ll\left(\frac{D^{2} \nu}{\epsilon}\right)^{-1 / 4},
$$

by studying the deformation of a single Fourier component in a homogeneous strain field. The sense of these results is that if $D \ll \nu$ the spectrum drops rapidly like $k^{-5 / 3}$, and then less rapidly like $k^{-1}$ before reaching an ultimate diffusive cutoff, while if $D=O(\nu)$, the cutoff occurs before the $k^{-1}$ range begins. There is experimental ${ }^{16,17}$ and some limited direct-numerical-simulation ${ }^{18}$ support for these results.

In the present paper we consider the solution of the passive-scalar equation in the presence of a velocity field of the stretched-spiral vortex type. In Sec. II we consider the dynamics of slender stretched vortices, and obtain the generic scalar diffusion equation in stretched space and time coordinates satisfied by both the axial velocity and the passive scalar. The solution of the generic equation by a twotime analysis is described in Sec. III, and examples are discussed consisting of the interaction of a diffusing line vortex with a shear flow, with and without the presence of an external stretching strain field. The spectrum associated with the spiral-like axial velocity distribution is obtained in Sec. V. When the vortex is stretched, a $k^{-7 / 3}$ spectrum is found at large wave number. This is produced by the axisymmetric part of the axial vorticity field and is subdominant to the $k^{-5 / 3}$ spectrum of the velocity induced by the axial vorticity.

The physical passive scalar problem is considered in Sec. VI. We picture the physical situation to be similar to the experiments of Gibson and Schwartz, ${ }^{16}$ in which nearly isotropic turbulence is created by flow through a grid. In these experiments, a passive scalar was injected into this flow through holes in the grid, as heated water or saline solution. We suppose that vortices are created at the grid and that scalar is injected into them at the creation. Both are stretched by the same large-scale motions. Differential rotation distorts the scalar into spiral bands, as for the axial velocity. We will find that the axially symmetric part of the velocity produces a $k^{-1}$ spectrum. The effect of the nonaxisymmetric, spiral part of the axial vorticity structure on the scalar evolution is found to produce a $k^{-5 / 3}$ component of the scalar spectrum.

\section{AXIAL MOTION AND SCALAR TRANSPORT IN THE STRETCHED SPIRAL VORTEX}

We consider a vortex embedded in a background linear velocity field. Denote vortex-fixed axes by $x_{i}$ with $x_{3}$ aligned with the vortex axis. Attention is restricted to a class of motions for which, at time $t=0$, all quantities of physical interest associated with the vortex motion are functions only of the cross-sectional coordinates $x_{2}, x_{3}$. Initial conditions of this type, when subject to a linear field have been studied extensively. ${ }^{1,10-13}$ Under fairly general conditions it can be shown ${ }^{19}$ that the initial independence of vortex velocities and vorticities on $x_{3}$ are preserved by the subsequent evolution in a frame of reference that rotates with angular velocity determined by components of the linear velocity. Subsequently, we will say that structures described by such fields, which also have compact support in the $x_{1}, x_{2}$ plane, exhibit cylindrical symmetry.

The Navier-Stokes equations for the velocity $v_{i}$ and the vorticity $\omega_{i}$ are

$$
\begin{aligned}
& \frac{\partial v_{i}}{\partial t}+v_{j} \frac{\partial v_{i}}{\partial x_{j}}=\frac{\partial P}{\partial x_{j}}+\nu \nabla^{2} v_{i}, \\
& \frac{\partial \omega_{i}}{\partial t}+v_{j} \frac{\partial \omega_{i}}{\partial x_{j}}=\omega_{j} \frac{\partial v_{i}}{\partial x_{j}}+\nu \nabla^{2} \omega_{i},
\end{aligned}
$$

where $P$ is the pressure-density ratio and $\nu$ is the kinematic viscosity. The velocity field is decomposed as

$$
v_{i}=u_{i}\left(x_{1}, x_{2}, t\right)+a_{i}(t) x_{i},
$$

with $a_{1}+a_{2}+a_{3}=0$ and $a_{3}>a_{2}>a_{1}$. In (5) the Einstein summation convection is not implied. If the support of $\omega_{i}(t)$ is compact in a domain surrounding $x_{1}=x_{2}=0$, then Eq. (5) corresponds to a vortex embedded in a pure background strain field whose eigenvector of principal strain is aligned with the $x_{3}$ direction. The velocity $u_{i}$ can then be expressed in terms of a vector potential $\psi_{i}\left(x_{1}, x_{2}, t\right)$ as

$$
u_{1}=\frac{\partial \psi_{3}}{\partial x_{2}}, \quad u_{2}=-\frac{\partial \psi_{3}}{\partial x_{1}}, \quad u_{3}=\frac{\partial \psi_{2}}{\partial x_{1}}-\frac{\partial \psi_{1}}{\partial x_{2}} .
$$

Choosing the gauge of $\psi_{i}$ such that $\partial \psi_{i} / \partial x_{i}=0$, it follows that

$$
\omega_{i}\left(x_{1}, x_{2}, t\right)=-\nabla_{2}^{2} \psi_{i}, \quad \nabla_{2}^{2} \equiv \frac{\partial^{2}}{\partial x_{1}^{2}}+\frac{\partial^{2}}{\partial x_{2}^{2}} .
$$

The axial motion is associated with $\psi_{1}, \psi_{2}$ while that in the $x_{1}, x_{2}$ plane is described by $\psi_{3}$.

When $P$ is written in the form

$$
P=P^{*}\left(x_{1}, x_{2}, t\right)-\frac{1}{2}\left(a_{1}^{2} x_{1}^{2}+a_{2}^{2} x_{2}^{2}+a_{3}^{2} x_{3}^{2}\right),
$$

where $P^{*}$ is a reduced pressure, using (5) and (6), Eqs. (3) and (4) for the components $\omega_{3}$ and $u_{3}$ can be written 


$$
\begin{aligned}
\frac{\partial \omega_{3}}{\partial t} & +\left(a_{1} x_{1}+\frac{\partial \psi_{3}}{\partial x_{2}}\right) \frac{\partial \omega_{3}}{\partial x_{1}}+\left(a_{2} x_{2}-\frac{\partial \psi_{3}}{\partial x_{1}}\right) \frac{\partial \omega_{3}}{\partial x_{2}} \\
& =a_{3} \omega_{3}+\nu \nabla_{2}^{2} \omega_{3}, \\
\frac{\partial u_{3}}{\partial t} & +\left(a_{1} x_{1}+\frac{\partial \psi_{3}}{\partial x_{2}}\right) \frac{\partial u_{3}}{\partial x_{1}}+\left(a_{2} x_{2}-\frac{\partial \psi_{3}}{\partial x_{1}}\right) \frac{\partial u_{3}}{\partial x_{2}}+a_{3} u_{3} \\
& =\nu \nabla_{2}^{2} u_{3},
\end{aligned}
$$

where we have assumed that the strain rates $a_{i}$ are constant in time. The equation governing the convection diffusion of a passive scalar field $c\left(x_{1}, x_{2}, t\right)$ by the vortex motion is

$$
\frac{\partial c}{\partial t}+\left(a_{1} x_{1}+\frac{\partial \psi_{3}}{\partial x_{2}}\right) \frac{\partial c}{\partial x_{1}}+\left(a_{2} x_{2}-\frac{\partial \psi_{3}}{\partial x_{1}}\right) \frac{\partial c}{\partial x_{2}}=D \nabla_{2}^{2} c,
$$

where $D$ is the molecular diffusion coefficient. It can be seen that (7) $(i=3)$ and (9) give two equations sufficient to determine $\psi_{3}\left(x_{1}, x_{2}, t\right)$ and $\omega_{3}\left(x_{1}, x_{2}, t\right)$. When these are solved, Eqs. (10) and (11) can then be solved for $u_{3}\left(x_{1}, x_{2}, t\right)$ and $c\left(x_{1}, x_{2}, t\right)$, respectively.

We now restrict attention to an axisymmetric strain field, $a_{1}=a_{2}=-a / 2, a_{3}=a, a>0$, for which case it is more convenient to work in polar coordinates $(r, \theta)$ with $x_{1}$ $=r \cos \theta, x_{2}=r \sin \theta$, and introduce the transformation ${ }^{1}$

$$
\begin{aligned}
& \rho=S(t)^{1 / 2} r, \quad \tau(t)=\int_{0}^{t} S\left(t^{\prime}\right) d t^{\prime}, \\
& S(t)=\exp \left(\int_{0}^{t} a\left(t^{\prime}\right) d t^{\prime}\right), \\
& \psi_{3}(r, \theta, t)=\widetilde{\psi}_{3}(\rho, \theta, \tau), \\
& \omega_{3}(r, \theta, t)=S(t) \tilde{\omega}_{3}(\rho, \theta, \tau), \\
& u_{3}(r, \theta, t)=S(t)^{-1} U_{3}(\rho, \theta, \tau), \\
& c(r, \theta, t)=C(\rho, \theta, \tau) .
\end{aligned}
$$

Equations (10) and (11) can then be expressed in essentially the same form

$$
\frac{\partial \phi}{\partial t}+\frac{1}{\rho}\left(\frac{\partial \widetilde{\psi}_{3}}{\partial \theta} \frac{\partial \phi}{\partial \rho}-\frac{\partial \widetilde{\psi}_{3}}{\partial \rho} \frac{\partial \phi}{\partial \theta}\right)=\kappa \nabla_{2}^{2} \phi
$$

where $(\phi, \kappa)$ are either $\left(U_{3}, \nu\right)$ or $(C, D)$. This is the scalar transport equation in the two-dimensional flow defined by $\widetilde{\psi}_{3}(\rho, \theta, \tau)$. Its solution, for given $\widetilde{\psi}_{3}(r, \theta, t)$ and appropriate initial conditions, will give the evolution of either the scalar or the axial velocity as the vortex winds up. ${ }^{10,13}$ Solutions for $u_{3}$ and $c$ will differ because their initial fields are not, in general, the same and second, because of the $S^{-1}$ factor in (15). The stream functions $\psi_{3}$ and $\widetilde{\psi}_{3}$ coincide at $t=0$ but develop differently when $t>0$ owing to the external strain. The same is true of $c, C$ and $u_{3}, U_{3}$. Since the strain rate $a$ is constant, then $S(t)=\exp (a t)$ and $\tau(t)=(S(t)-1) / a$.

\section{SOLUTION OF THE PASSIVE SCALAR EQUATION}

We now consider the solution of (17). The velocity field is assumed to be given by an approximate solution of the
Navier-Stokes equations corresponding to the stretched spiral vortex. ${ }^{1}$ In this solution, differential rotation within the vortex deforms the nonaxially symmetric part of the vorticity into spiraling vortex layers. The solution for the vorticity and the stream function takes the form

$$
\begin{array}{ll}
\tilde{\omega}_{3}=\sum_{-\infty}^{\infty} \omega_{n}(\rho, \tau) \exp (\operatorname{in} \theta), & \omega_{-n}=\omega_{n}^{*}, \\
\widetilde{\psi}_{3}=\sum_{-\infty}^{\infty} \psi_{n}(\rho, \tau) \exp (\operatorname{in} \theta), \quad \psi_{-n}=\psi_{n}^{*},
\end{array}
$$

where the Fourier coefficients, for $n \neq 0$, are

$$
\begin{aligned}
\omega_{n}(\rho, \tau)= & f_{n}(\rho) \exp (-i n \Omega(\rho) \tau) \\
& \times \exp \left(-\nu n^{2} \Lambda^{2} \tau^{3} / 3\right), \\
\psi_{n}(\rho, \tau)= & \tau^{-2} h_{n}(\rho) \exp (-i n \Omega(\rho) \tau) \\
& \times \exp \left(-\nu n^{2} \Lambda^{2} \tau^{3} / 3\right),
\end{aligned}
$$

with

$$
h_{n}(\rho)=\frac{f_{n}(\rho)}{n^{2} \Lambda^{2}}, \quad \Lambda(\rho)=\frac{d \Omega(\rho)}{d \rho} .
$$

The local angular velocity $\Omega(\rho)$ is related to the zeroth harmonic of the vorticity (circle-averaged vorticity) and to $\psi_{0}$ as

$$
\omega_{0}(\rho)=\frac{1}{\rho} \frac{\partial\left(\rho^{2} \Omega\right)}{\partial \rho}, \quad \Omega(\rho)=-\frac{1}{\rho} \frac{\partial \psi_{0}}{\partial \rho} .
$$

It is easily verified that (18)-(23) satisfies $\nabla_{2}^{2} \widetilde{\psi}_{3}=-\widetilde{\omega}_{3}$ to $O\left(\tau^{-2}\right)$. In the above it is assumed that $\Lambda<0$. The functions $f_{n}$ are arbitrary "fictitious" initial conditions that define a spiral vortex structure. If these functions are independent of $n$ the structure can be thought of as a vortex sheet rolling up. The above solution is asymptotic in time. It essentially describes the nonaxisymmetric component of a vorticity field being wound by the axisymmetric part. The nonaxisymmetric part of the velocity field becomes small as the vorticity is wound around the core but the nonaxisymmetric vorticity may not be small except through viscous diffusion.

The problem is now to solve the scalar equation (17) with $\widetilde{\psi}_{3}$ given by (19). It is natural to seek solutions in the form of inverse powers of $\tau$. We first write (19) in the form

$$
\begin{aligned}
& \widetilde{\psi}_{3}=\widetilde{\psi}^{(0)}+\tau^{-2} \widetilde{\psi}^{(2)}, \\
& \widetilde{\psi}^{(2)}=\sum_{-\infty, n \neq 0}^{\infty} \widetilde{\psi}_{n}^{(2)} \exp (i n(\theta-\Omega \tau)), \\
& \widetilde{\psi}_{n}^{(2)}=h_{n}(\rho) \exp \left(-\nu n^{2} \Lambda^{2} \tau^{3} / 3\right) .
\end{aligned}
$$

Next, we introduce the Lagrangian variable $\alpha=\theta-\Omega \tau$ so that

$$
\frac{\partial}{\partial \theta} \rightarrow \frac{\partial}{\partial \alpha}, \quad \frac{\partial}{\partial \rho} \rightarrow \frac{\partial}{\partial \rho}-\Lambda \tau \frac{\partial}{\partial \alpha}, \quad \frac{\partial}{\partial \tau} \rightarrow \frac{\partial}{\partial \tau}-\Omega \frac{\partial}{\partial \alpha} .
$$

Equation (17) then becomes

$$
\frac{\partial \phi}{\partial \tau}-\kappa \nabla^{2} \phi=\tau^{-2} \frac{1}{\rho}\left(\frac{\partial \widetilde{\psi}^{(2)}}{\partial \rho} \frac{\partial \phi}{\partial \alpha}-\frac{\partial \widetilde{\psi}^{(2)}}{\partial \alpha} \frac{\partial \phi}{\partial \rho}\right),
$$


where $\nabla^{2}$ is now written

$$
\nabla^{2}=\left(\frac{\partial}{\partial r}-\Lambda \tau \frac{\partial}{\partial \alpha}\right)^{2}+\frac{1}{\rho}\left(\frac{\partial}{\partial r}-\Lambda \tau \frac{\partial}{\partial \alpha}\right)+\frac{1}{\rho^{2}} \frac{\partial^{2}}{\partial \alpha^{2}} .
$$

This will be approximated, for large $\tau$, by the term proportional to $\tau^{2}$. Thus the scalar equation becomes

$$
\frac{\partial \phi}{\partial \tau}-\kappa(\Lambda \tau)^{2} \frac{\partial^{2} \phi}{\partial \alpha^{2}}=\tau^{-2} \frac{1}{\rho}\left(\frac{\partial \widetilde{\psi}^{(2)}}{\partial \rho} \frac{\partial \phi}{\partial \alpha}-\frac{\partial \widetilde{\psi}^{(2)}}{\partial \alpha} \frac{\partial \phi}{\partial \rho}\right) .
$$

The form of the left-hand side of this equation shows that diffusion introduces a slow time variable $T_{\kappa}=\left(\kappa \Lambda^{2}\right)^{1 / 3} \tau$. This can be estimated to be of order $\left(\kappa / \Gamma_{0}\right)^{1 / 3} \Omega_{0} \tau$, where $R$ and $\Omega_{0}$ are characteristic length and inverse time scales associated with the axisymmetric part of the motion due to the axial vorticity [Eqs. (23)], and $\Gamma_{0} \sim R^{2} \Omega_{0}$ is a measure of the axial circulation around a typical vortex, estimated ${ }^{4,5}$ as $\Gamma_{0} / \nu=O\left(10^{3}\right)$. The ratio of the fast time scale $\Omega_{0} \tau$ associated with particle transit around the vortex to the slow time scale is then $O\left(\Gamma_{0} / \kappa\right)^{1 / 3}=O(\operatorname{ReSc})^{1 / 3}$, where $\operatorname{Re}=\Gamma_{0} / \nu$ is the vortex Reynolds number and $\mathrm{Sc}=\nu / \kappa$ is the Schmidt number. The $\mathrm{Re}^{1 / 3}$, scaling is typically seen in swirling vortex flows. ${ }^{1,11,12,20}$ When Re $\gg 1$ and $\kappa=O(\nu)$ or smaller, the slow time scale is small compared with the particle transit time, and consequently diffusion is a much slower process than differential rotation and $\left(\kappa \Lambda^{2}\right)^{1 / 3}$ can be treated as a small parameter.

A solution to (30) is now found by a two-time analysis in the form of a series in inverse powers of the fast time with coefficients which are functions of $\rho$ and the slow time. First, using

$$
\frac{\partial \phi\left(\tau, T_{\kappa}\right)}{\partial \tau}=\left(\frac{\partial \phi}{\partial \tau}\right)_{T_{\kappa}}+\left(\kappa \Lambda^{2}\right)^{1 / 3}\left(\frac{\partial \phi}{\partial T_{\kappa}}\right)_{\tau},
$$

Eq. (30) is written as

$$
\begin{gathered}
\left(\frac{\partial \phi}{\partial \tau}\right)_{T_{\kappa}}+\left(\kappa \Lambda^{2}\right)^{1 / 3}\left[\left(\frac{\partial \phi}{\partial T_{\kappa}}\right)_{\tau}-T_{\kappa}^{2} \frac{\partial^{2} \phi}{\partial \alpha^{2}}\right] \\
=\tau^{-2} \frac{1}{\rho}\left(\frac{\partial \widetilde{\psi}^{(2)}}{\partial \rho} \frac{\partial \phi}{\partial \alpha}-\frac{\partial \widetilde{\psi}^{(2)}}{\partial \alpha} \frac{\partial \phi}{\partial \rho}\right) .
\end{gathered}
$$

We expand (32) in a formal series in powers of the small parameter $\left(\kappa \Lambda^{2}\right)^{1 / 3}$. The first term, independent of this parameter, satisfies (32) without the bracketed term on the left. This is solved in the form

$$
\phi=\phi^{(0)}+\tau^{-1} \phi^{(1)}+\cdots .
$$

We find $\phi^{(0)}$ is an arbitrary function of $\rho, T_{\kappa}, \alpha$, and $\phi^{(1)}$ is given by

$$
\phi^{(1)}=-\frac{1}{\rho}\left(\frac{\partial \widetilde{\psi}^{(2)}}{\partial \rho} \frac{\partial \phi^{(0)}}{\partial \alpha}-\frac{\partial \widetilde{\psi}^{(2)}}{\partial \alpha} \frac{\partial \phi^{(0)}}{\partial \rho}\right)
$$

and would be completely determined if $\phi^{(0)}$ were known. The next term in the $\left(\kappa \Lambda^{2}\right)^{1 / 3}$ expansion satisfies

$$
\begin{gathered}
\left(\frac{\partial \phi_{1}}{\partial \tau}\right)_{T_{\kappa}}+\left[\left(\frac{\partial \phi^{(0)}}{\partial T_{\kappa}}\right)_{\tau}-T_{\kappa}^{2} \frac{\partial^{2} \phi^{(0)}}{\partial \alpha^{2}}\right] \\
=\tau^{-2} \frac{1}{\rho}\left(\frac{\partial \widetilde{\psi}^{(2)}}{\partial \rho} \frac{\partial \phi_{1}}{\partial \alpha}-\frac{\partial \widetilde{\psi}^{(2)}}{\partial \alpha} \frac{\partial \phi_{1}}{\partial \rho}\right),
\end{gathered}
$$

which we again solve in a series in inverse powers of $\tau$. But the first term will be a secular term, linear in $\tau$, unless its coefficient is zero. Therefore $\phi^{(0)}$ must satisfy

$$
\left(\frac{\partial \phi^{(0)}}{\partial T_{\kappa}}\right)_{\tau}=T_{\kappa}^{2} \frac{\partial^{2} \phi^{(0)}}{\partial \alpha^{2}}
$$

We then solve (34) and (36) in the form

$$
\begin{aligned}
& \phi^{(0)}=\sum_{-\infty}^{\infty} \phi_{n}^{(0)}\left(\rho, T_{\kappa}\right) \exp (\text { in } \alpha), \\
& \phi^{(1)}=\sum_{-\infty, n \neq 0}^{\infty} \phi_{n}^{(1)}\left(\rho, T_{\kappa}\right) \exp (i n \alpha),
\end{aligned}
$$

with the results

$$
\phi_{n}^{(0)}(\rho, \tau)=\hat{\phi}_{n}^{(0)}(\rho) \exp \left(-\kappa n^{2} \Lambda^{2} \tau^{3} / 3\right)
$$

where the $\hat{\phi}_{n}^{(0)}(\rho)$ define the initial structure of the $\phi$ field, and

$\phi_{n}^{(1)}=\frac{i}{\rho} \sum_{-\infty, m \neq 0}^{\infty}\left(m \widetilde{\psi}_{m}^{(2)} \frac{\partial \phi_{n-m}^{(0)}}{\partial \rho}-(n-m) \frac{\partial \widetilde{\psi}_{m}^{(2)}}{\partial \rho} \phi_{n-m}^{(0)}\right)$,

where $\widetilde{\psi}_{n}^{(2)}$ are given by (26). Equations (37) and (39) give a well-known approximate solution ${ }^{21,22}$ to (17) when the velocity field is given by the axisymmetric part of the vorticity field. For the axial velocity $(\phi, \kappa) \equiv\left(U_{3}, \nu\right)$, the slow time scale is the same as that for the radial diffusion of axial vorticity, as given in $(20)$. When $(\phi, \kappa) \equiv(C, D)$, the ratio of the diffusion time scale for the scalar to that for the vorticity is $T_{D} / T_{\nu}=O\left(\mathrm{Sc}^{-1 / 3}\right)$.

\section{EVOLUTION OF THE AXIAL VELOCITY}

The evolution of the axial motion can now be obtained by putting $(\phi, \kappa) \equiv\left(U_{3}, \nu\right)$ in the above, and using $(15)$. We thus write, for the evolution of the axial velocity in the spiral vortex

$u_{3}(r, \theta, t)=S(t)^{-1} U_{3}(\rho, \theta, \tau)$

$U_{3}=U^{(0)}+\tau^{-1} \sum_{-\infty, n \neq 0}^{\infty} U_{n}^{(1)}(\rho, \tau) \exp (i n(\theta-\Omega \tau))$,

$U^{(0)}=\sum_{-\infty}^{\infty} U_{n}^{(0)} \exp (i n(\theta-\Omega \tau))$

$U_{n}^{(0)}=\hat{U}_{n}^{(0)}(\rho) \exp \left(-\nu n^{2} \Lambda^{2} \tau^{3} / 3\right)$,

$U_{n}^{(1)}=\frac{i}{\rho} \sum_{-\infty, m \neq 0}^{\infty}\left(m \widetilde{\psi}_{m}^{(2)} \frac{\partial U_{n-m}^{(0)}}{\partial \rho}-(n-m) \frac{\partial \widetilde{\psi}_{m}^{(2)}}{\partial \rho} U_{n-m}^{(0)}\right)$, 
where the initial axial velocity field is given by the functions $\hat{U}_{n}^{(0)}(\rho)$. The $r$ and $\theta$ components of the vorticity are

$$
\omega_{r}=\frac{1}{r} \frac{\partial u_{3}}{\partial \theta}, \quad \omega_{\theta}=-\frac{\partial u_{3}}{\partial r},
$$

from which, using (41)-(45)

$$
\begin{aligned}
& \omega_{r}=\frac{1}{S^{1 / 2}} \sum_{-\infty}^{\infty} A_{n} \exp (i n \theta)+O\left(\tau^{-1}\right), \\
& \omega_{\theta}=\frac{\tau}{S^{1 / 2}} \sum_{-\infty}^{\infty} B_{n} \exp (i n \theta)+O\left(\tau^{-1 / 2}\right), \\
& A_{n}=\frac{i n}{\rho} U_{n}^{(0)} \exp (-i n \Omega \tau) \\
& B_{n}=i n \Lambda U_{n}^{(0)} \exp (-i n \Omega \tau)
\end{aligned}
$$

It can be seen that the leading-order terms for both components of the vorticity normal to the vortex axis are functions only of the Fourier coefficients $\hat{U}_{n}^{(0)}$ of the initial axial velocity distribution and of the structure of the vortex core. It will be recalled that $S(t) \sim a \tau$ when $\tau$ is large. Hence $\omega_{\theta}$ $\gg \omega_{r}$.

\section{A. Diffusing line vortex in a shear flow: $a=0$}

As an example of the above solution, we consider the interaction of a diffusing line vortex with a background shear flow aligned such that at $t=0$ the shear-flow streamlines are parallel to the vortex filament. ${ }^{10-12}$ We first take $a=0$ with initial axial velocity field

$$
\hat{U}^{(0)}=\omega_{0} r \sin \theta,
$$

where $\omega_{0}$ is the shear-flow vorticity. For the diffusing line vortex the function $\Omega$ takes the form

$$
\Omega=\frac{\Gamma_{0}}{2 \pi r^{2}}\left(1-\exp \left(-\frac{r^{2}}{4 \nu t}\right)\right)
$$

where $\Gamma_{0}$ is the vortex circulation. The azimuthal vorticity is

$$
\frac{\omega_{\theta}}{\omega_{0}}=(r \Lambda t \cos (\theta-\Omega t)-\sin (\theta-\Omega t)) e^{-\nu \Lambda^{2} t^{3} / 3},
$$

where we have included only the terms of $O(1)$. A solution close to this form was obtained by Kawahara et al. ${ }^{12}$ It is a function only of $\operatorname{Re}=\Gamma_{0} / 2 \pi \nu$ and the similarity variable $\eta$ $=r / 2(\nu t)^{1 / 2}$. Figure 1 shows the maximum azimuthal vorticity on a circle, $\left|\omega_{\theta} / \omega_{0}\right|$, for $\operatorname{Re}=1000$ compared to an asymptotic expression, valid for $\eta \mathrm{Re}^{-1 / 4} \gg 1$, obtained by Moore. ${ }^{11}$ It can be shown from (51) and (52) that, when $\mathrm{Re}^{1 / 3} \gg 1$, the maximum dimensionless vorticity is $0.903 \mathrm{Re}^{1 / 3}$ at a radius $r=2^{1 / 3}(\nu t)^{1 / 2} \mathrm{Re}^{1 / 3}$ in close agreement with the exact results. ${ }^{10,11}$ Figure 1 shows the similarity azimuthal vorticity variation along the $y$ axis. As the line vortex diffuses, its swirling velocity field winds the vorticity associated with the shear flow of order $(\nu / t)^{1 / 2} \operatorname{Re}^{1 / 3}$ and there is an energy transfer to larger scales at time progresses. The peak azimuthal vorticity achieves a value determined by a balance between azimuthal vortex stretching and viscous diffusion.

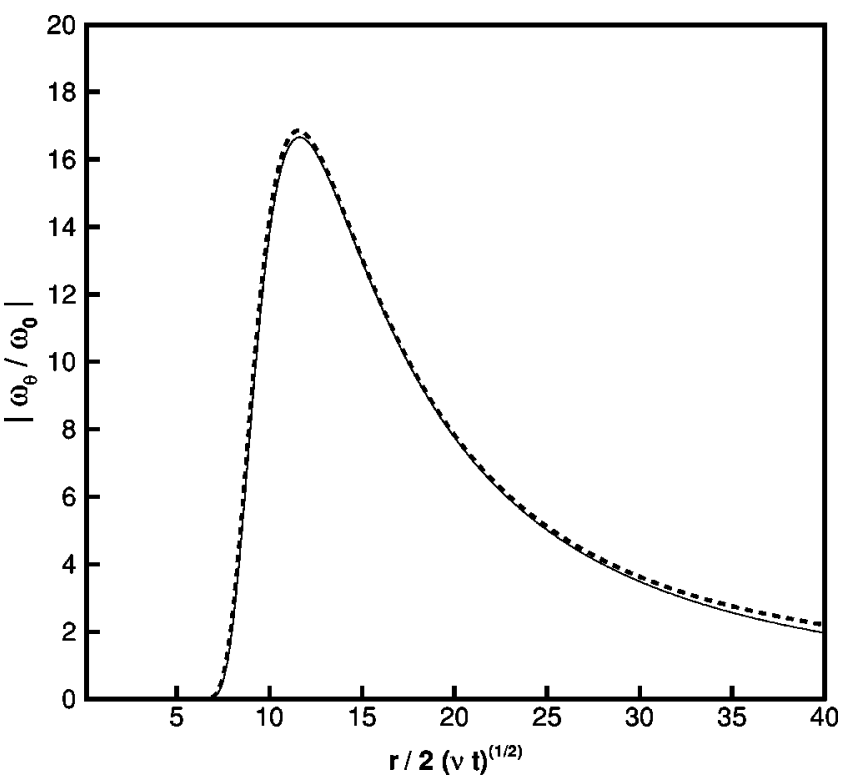

FIG. 1. Maximum vorticity in diffusing line vortex aligned with a shear flow. $\operatorname{Re}=1000$. Solid, present. Dotted, Moore (Ref. 11).

\section{B. Diffusing line vortex in a shear flow: $a>0$}

For positive nonzero strain $a>0$, the above solution holds in stretched coordinates $(\rho, \tau)$. In unstretched $(r, t)$ variables

$\frac{\omega_{\theta}}{\omega_{0}}=(\rho \Lambda \tau \cos (\theta-\Omega \tau)-\sin (\theta-\Omega \tau)) e^{-\nu \Lambda^{2} \tau^{3} / 3-a t / 2}$,

where $\rho(r, t)$ and $\tau(t)$ are given by (12). The maximum vorticity is $0.903 \mathrm{Re}^{1 / 3} \exp (-a t)$ at radius $r$ $=2^{1 / 3}(\nu / a)^{1 / 2} \operatorname{Re}^{1 / 3}$. When $a>0$ the solution is not of similarity form. The outward movement of the spiral structure is inhibited by the radial velocity associated with the strain field.

\section{ENERGY SPECTRUM CONTRIBUTED BY THE AXIAL VELOCITY}

Lundgren ${ }^{1}$ showed how to calculate the energy spectrum associated with the axial vorticity $\omega_{3}$ for a statistical ensemble of cylindrical vortex structures. This can be generalized to define the shell-integrated spectra of other physical quantities inside cylindrical vortices. ${ }^{2,5,6}$ Briefly, a correlation function for some function $\Phi(r, \theta, t)$ is first defined inside a cylindrical vortex subject to axial strain of the type previously described, and this is then converted to a corresponding energy spectrum by taking the appropriate Fourier transform. When this is integrated over a spherical shell of wave number $k$ in wave number space, and account is taken of the cylindrical vortex structure, an expression for the instantaneous spectrum of $\Phi$ results in terms of its Fourier coefficients in a Fourier expansion

$$
\Phi(r, \theta, t)=\sum_{-\infty}^{\infty} \Phi(r, t)_{n} \exp (\text { in } \theta) .
$$


The instantaneous spectrum is distinguished from that of an ensemble of structures by first supposing that a box of side $L$ is populated at time $t$ by a collection of such vortices. These structures, each of initial length $l_{0}$, undergo an identical evolution from different origins of time. Structures are created at a rate $N_{c}$ per unit time by some external process not described by the model. At time $t$ the total turbulent field is the superposition of the contribution of each structure, at its stage on the evolution curve. A statistical equilibrium is envisioned, whereby structures are created, stretched by the strain provided by larger scales, and finally decay by internal radial diffusion. For details, see Lundgren ${ }^{1}$ or Pullin and Saffman. ${ }^{7}$

The resulting spectrum of $\Phi$ for the ensemble of structures is then the sum of the contributions of the individual members of the ensemble. Since all structures undergo the same evolution, the sum over the ensemble can be replaced by an integral over the lifetime of a typical structure (ergodic hypothesis). It is found that

$$
\begin{aligned}
E_{\Phi}(k)= & 2 \pi N k \int_{t_{1}}^{t_{2}} S(t) \\
& \times\left(I_{0}(r, t) I_{0}^{*}(r, t)+\sum_{1}^{\infty}\left|I_{n}(r, t)\right|^{2}\right) d t, \\
I_{n}(r, t)= & \int_{0}^{\infty} J_{n}(k r) \Phi_{n}(r, t) r d r,
\end{aligned}
$$

where $N=N_{c} l_{0} / L^{3}, t_{1}-t_{2}$ is the structure lifetime and $J_{n}$ is the $J$-Bessel function of order $n$. The factor $S(t)$ in the integrand accounts for the vortex stretching by the external strain, whereby the vortex length at time $t$ is $l(t)=l_{0} S(t)$. If there is more than one type of vortex, each with its own internal structure and evolution, then (56) can be extended to a sum over the vortex types.

\section{A. The axial velocity spectrum: $a>0$}

That part of the energy spectrum contributed by axial motion can be obtained from (15), (42)-(45) and (55) and (56), with $\Phi$ replaced by $u_{3}$ in (55). We presently follow an alternative but equivalent method which better reveals the relation of the axial-velocity spectrum to the azimuthal and radial vorticity. These two methods give identical results. For the cylindrical structures presently studied, the threedimensional energy (velocity) spectrum $E(k)$ for homogeneous, but not necessarily isotropic turbulence, can be expressed as

$$
\begin{aligned}
E(k)= & E_{\omega_{r}}(k)+E_{\omega_{\theta}}+E_{\omega_{3}}(k) \\
= & \frac{\mathcal{E}_{\omega_{r}}(k)+\mathcal{E}_{\omega_{\theta}}(k)+\mathcal{E}_{\omega_{3}}(k)}{2 k^{2}},
\end{aligned}
$$

where $\mathcal{E}_{\omega_{r}}(k), \mathcal{E}_{\omega_{\theta}}(k)$, and $\mathcal{E}_{\omega_{3}}(k)$ are the three-dimensional vorticity spectra associated with the components of vorticity indicated and $E_{\omega_{r}}(k), E_{\omega_{\theta}}(k), E_{\omega_{3}}(k)$ are corresponding parts of the velocity spectrum. The vorticity spectra correspond to correlations of vorticity components within the cy- lindrical vortex structure in the sense described above. The component $E_{\omega_{3}}(k)$ has been calculated for $\omega_{3}$ given by (14) and (18) with the result ${ }^{1}$

$$
\begin{aligned}
E_{\omega_{3}}(k)= & E_{0}(k)+\frac{4 \pi}{3} N a^{1 / 3} k^{-5 / 3} \exp \left[-\frac{2 \nu k^{2}}{3 a}\right] \\
& \times \sum_{n=1}^{\infty} n^{-4 / 3} \int_{0}^{\infty} \frac{\left|f_{n}(\rho)\right|^{2} \rho d \rho}{|\Lambda(\rho)|^{4 / 3}}
\end{aligned}
$$

where $E_{0}(k) \sim k^{-1}$ is the spectrum of the axisymmetric core. We now obtain $\mathcal{E}_{\omega_{\theta}}(k)$ and subsequently $E_{\omega_{\theta}}$. It will be shown that this is the dominant contribution from the nonaxial vorticity, when $k$ is large, and that the velocity spectrum associated with $\omega_{r}$, and with higher order corrections to the vorticity in (47) and (48) give subdominant terms.

Attention is restricted to axial velocity without the zeroth harmonic, so that $\hat{U}_{0}^{(0)} \equiv 0$. We first consider the case $a>0$. The spectrum of $\omega_{\theta}$ can be calculated by replacing the $\Phi_{n}$ in (56) by the equivalent Fourier coefficients in (48). Using, from (12), that $r d r=\rho d \rho / S(t)$, the integrals (56) then take the form

$$
\begin{aligned}
I_{n}(r, t)= & \frac{1}{S} \int_{0}^{\infty} J_{n}\left(\frac{k \rho}{S^{1 / 2}}\right) \hat{B}_{n}(\rho, \tau) \rho d \rho, \quad n \neq 0, \\
\hat{B}_{n}(\rho, \tau)= & \frac{i \tau}{S^{1 / 2}} n \Lambda(\rho) \hat{U}_{n}^{(0)}(\rho) \\
& \times \exp \left(-i n \Omega(\rho) \tau-\nu n^{2} \Lambda^{2}(\rho) \tau^{3} / 3\right),
\end{aligned}
$$

and $I_{0}=0$. The integral can be approximated for large $k$ by using the asymptotic expansion for the Bessel function

$$
\begin{aligned}
J_{n}(k r) \approx & \frac{1}{2}\left(\frac{2}{\pi k r}\right)^{1 / 2}\left((-i)^{n+1 / 2} \exp (i k r)\right. \\
& \left.+(i)^{n+1 / 2} \exp (-i k r)\right) .
\end{aligned}
$$

Use of (62) in (60) produces factors of the form $\exp \left[i k \rho / S^{1 / 2} \pm i n \Omega(\rho) \tau\right]$, which are rapidly varying functions of $\rho$ when $k$ and $\tau$ are large. The integral is then of the type that can be estimated by the method of stationary phase. The principal contribution comes from a region surrounding the point of stationary phase, given by a solution, $\rho_{n}$, of

$$
k+n S^{1 / 2} \Lambda\left(\rho_{n}\right) \tau=0 .
$$

This gives, after some algebra

$$
\begin{aligned}
I_{n} I_{n}^{*} \approx & \frac{\rho_{n} \tau n \Lambda(\rho)^{2}\left|\hat{U}_{n}^{(0)}\left(\rho_{n}\right)\right|^{2}}{S^{3 / 2} \Lambda^{\prime}\left(\rho_{n}\right) k} \\
& \times \exp \left(-2 \nu n^{2} \Lambda^{2}\left(\rho_{n}\right) \tau^{3} / 3\right) .
\end{aligned}
$$

Equation (63) is a relation between $\tau$ and $\rho_{n}$ both in (64) and in the integral (55), when the latter is transformed to the integration variable $\tau$ over the interval $\left(\tau_{1}, \tau_{2}\right)$, using (12), with $\tau_{1}=\tau\left(t_{1}\right), \quad \tau_{2}=\tau\left(t_{2}\right)$. Using $S(\tau) \approx a \tau$ when $a \tau$ is large, Eq. (63) becomes, approximately

$$
\tau \approx\left(\frac{k}{n a^{1 / 2}\left|\Lambda\left(\rho_{n}\right)\right|}\right)^{2 / 3}
$$


Substitution of (65) into (64) gives algebraic simplifications. When this last result, Eq. (12), and (65) are substituted into (56) $\left(E_{\Phi} \rightarrow \mathcal{E}_{\omega_{\theta}}\right)$, and $E_{\omega_{\theta}}(k)=\mathcal{E}_{\omega_{\theta}}(k) / 2 k^{2}$ is used, we obtain

$$
\begin{aligned}
E_{\omega_{\theta}}(k)= & \frac{4 \pi N}{3 a^{7 / 3}} k^{-7 / 3} \exp \left(-\frac{2 k^{2} \nu}{3 a}\right) \sum_{n=1}^{\infty} n^{4 / 3} \\
& \times \int_{0}^{\infty}|\Lambda(\rho)|^{4 / 3}\left|\hat{U}_{n}^{(n)}(\rho)\right|^{2} \rho d \rho .
\end{aligned}
$$

In the above we have assumed that the interval $\left(\tau_{1}, \tau_{2}\right)$ can be replaced by $(0, \infty)$ and that the integral converges at these limits. The integral over $\rho$ is divergent when $\Omega$ is given by a point vortex flow and the initial axial velocity is specified by (50). When this same axial velocity is combined with other physically motivated choices ${ }^{3,4}$ for $\Omega$, the integral in (66) can be shown to converge at both limits.

It follows from (48) and (65) that higher-order terms in the azimuthal vorticity give a factor $k^{-2 / 3}$ in corrections to (66), when $k$ is large. Similarly, that part of $E(k)$ associated with the radial vorticity, $E_{\omega_{r}}(k)$, can be shown to be $O\left(k^{-3}\right)$ for large $k$. The velocity spectrum associated with the nonaxial vorticity is therefore produced mainly by axial motions generated by the leading-order azimuthal vorticity, but is subdominant in comparison with $E_{\omega_{3}}(k)$, given by (59), when $k$ is large.

The ratio of the mean-square azimuthal vorticity to the nonaxisymmetric part of the mean square axial vorticity is

$$
\begin{aligned}
\overline{\frac{\omega_{\theta}^{2}}{\overline{\omega_{3}^{2}}}} \equiv & \frac{\int_{0}^{\infty} k^{2} E_{\omega_{\theta}}(k) d k}{\int_{0}^{\infty} k^{2} E_{\omega_{3}}(k) d k} \\
& =\frac{2^{1 / 3} \Gamma[1 / 3] \nu^{1 / 3}}{\Gamma[2 / 3] a^{3}} \frac{\sum_{n=1}^{\infty} n^{4 / 3} \int_{0}^{\infty}|\Lambda(\rho)|^{4 / 3}\left|\hat{U}_{n}^{(0)}(\rho)\right|^{2} \rho d \rho}{\sum_{n=1}^{\infty} n^{-4 / 3} \int_{0}^{\infty}|\Lambda(\rho)|^{-4 / 3}\left|f_{n}(\rho)\right|^{2} \rho d \rho},
\end{aligned}
$$

where $\Gamma[\cdots]$ denotes the gamma function. To estimate this ratio we set

$$
\begin{aligned}
& f_{n}(\rho)=\frac{\Gamma_{0}}{R^{2}} \widetilde{f}_{n}(\xi), \quad \xi=\frac{\rho}{R}, \\
& \Omega(\rho)=\frac{\Gamma_{0}}{R^{2}} \widetilde{\Omega}(\xi), \quad \Lambda(\rho)=\frac{\Gamma_{0}}{R^{3}} \widetilde{\Lambda}(\xi), \\
& \hat{U}_{n}^{(0)}=u^{\prime} \tilde{\hat{U}}_{n}^{(0)},
\end{aligned}
$$

where $\Gamma_{0}, R$ are circulation and length scales associated with the axial vorticity, $u^{\prime}$ is an axial velocity scale, and the tilde used here denotes a dimensionless function. Using these relations in (67) and assuming that the resulting dimensionless integrals are of order unity gives

$$
\overline{\overline{\omega_{3}^{2}}} \sim \frac{\left(\Gamma_{0} / \nu\right)^{2 / 3}\left(u^{\prime} R / \nu\right)^{2}}{\left(a R^{2} / \nu\right)^{3}}
$$

Pullin et al. $^{5}$ discuss the scaling of parameters in the stretched spiral vortex in terms of the Taylor microscale $R_{\lambda}$.
They argue, on the basis of the coherence of strain along a vortex of finite length, that variations in axial velocity are limited by the root-mean-square velocity for the turbulence $\left(\overline{u_{i}^{2}}\right)^{1 / 2}$. With other assumptions this gives $R \sim \lambda$, where $\lambda$ is the Taylor microscale. If it is further argued that the local axial velocity scale is $u^{\prime}=O\left(\overline{u_{i}^{2}}\right)^{1 / 2}$, then $u^{\prime} R / \nu \sim O\left(\operatorname{Re}_{\lambda}\right)$. Using $^{5,4} \Gamma_{0} / \nu=10^{3}$ and estimates ${ }^{5}$ of $a R^{2} / \nu$ in (71) suggests $\overline{\omega_{\theta}^{2}} / \overline{\omega_{3}^{2}}=O(1)$ at $\operatorname{Re}_{\lambda} \approx 200$, which is typical of present direct numerical simulations. Different estimates can be obtained depending on scaling assumptions.

\section{B. The axial velocity spectrum: $a=0$}

Equation (66) is singular when $a \rightarrow 0$, and so the case with no axial stretching, $a=0$, requires separate treatment. The reason for this is the use of the approximation $S \approx a \tau$, $a \tau \gg 1$, which is not appropriate for $a=0$. The axial velocity spectrum can be obtained for this latter case by repeating the above analysis, replacing the stationary phase relationship (63) by

$$
k+n \Lambda(r) t=0
$$

The spectrum of the axial flow can then be obtained by repeating the preceding analysis, with the result

$$
\begin{aligned}
E_{\omega_{\theta}}(k)= & 2 \pi N \sum_{n=1}^{\infty} \frac{1}{n} \int_{0}^{\infty} \frac{r\left|\hat{U}_{n}^{(n)}(r)\right|^{2}}{|\Lambda(r)|} \\
& \times \exp \left(-\frac{2 \nu k^{3}}{3 n|\Lambda(r)|}\right) \rho d \rho .
\end{aligned}
$$

The dissipation is

$$
\epsilon=2 \nu \int_{0}^{\infty} k^{2} E_{\omega_{\theta}}(k) d k=2 \pi N \sum_{n=1}^{\infty} \int_{0}^{\infty}\left|\hat{U}_{n}^{(0)}(r)\right|^{2} r d r .
$$

This is, as expected, just the total kinetic energy contained in the initial axial velocity field. It can be seen that the righthand side of (73) combines functions of both $k$ and $r$ within the integral. Obtaining a definite spectrum for large $k$ then requires specific forms for $\Omega(r)$ and $\hat{U}_{n}^{(0)}(r)$. We choose $\Omega \sim \Gamma_{0} /\left(2 \pi r^{2}\right)$ and obtain $\hat{U}_{1}^{(0)}(r)$ from (50). Evaluating (73) then gives

$$
\begin{aligned}
& E_{\omega_{\theta}}(k)=\mathcal{B} N \Gamma_{0}^{4 / 3} \omega_{0}^{2} \nu^{-7 / 3} k^{-7}, \\
& \mathcal{B}=2^{-1}\left(\frac{3}{2 \pi}\right)^{1 / 3} \Gamma[4 / 3] .
\end{aligned}
$$

\section{SPECTRUM OF A PASSIVE SCALAR}

We now consider the spectrum of a passive scalar. The evolution of the passive scalar can be obtained by setting $(\phi, \kappa)=(C, D)$. This gives

$$
\begin{aligned}
c(r, \theta, t)= & c^{(0)}+\tau^{-1} \sum_{-\infty, n \neq 0}^{\infty} c_{n}^{(1)}\left(\rho, T_{\nu}, T_{D}\right) \\
& \times \exp (i n(\theta-\Omega \tau)),
\end{aligned}
$$




$$
\begin{aligned}
& c^{(0)}=\sum_{-\infty}^{\infty} c_{n}^{(0)}\left(\rho, T_{D}\right) \exp (i n(\theta-\Omega \tau)), \\
& c_{n}^{(0)}=\hat{c}_{n}^{(0)}(\rho) \exp \left(-D n^{2} \Lambda^{2} \tau^{3} / 3\right), \\
& c_{n}^{(1)}=\frac{i}{\rho} \sum_{-\infty, m \neq 0}^{\infty}\left(m \widetilde{\psi}_{m}^{(2)} \frac{\partial c_{n-m}^{(0)}}{\partial \rho}-(n-m) \frac{\partial \widetilde{\psi}_{m}^{(2)}}{\partial \rho} c_{n-m}^{(0)}\right),
\end{aligned}
$$

where the initial scalar field is given by the functions $\hat{c}_{n}^{(0)}(\rho)$ using

$$
c(r, \theta, 0)=\sum_{-\infty}^{\infty} \hat{c}_{n}^{(0)}(r) \exp (i n \theta)
$$

From (79) and (26), it follows that the coefficients $c_{n}^{(1)}$ are functions of the slow time scales for passive scalar and vorticity diffusion in the spiral. The spectrum of the passive scalar can now be obtained, mutatis mutantis, from the analysis leading to the spectrum of the axial velocity. On replacing $\Phi$ with $c$ in (55) and (56), and after considerable algebra, it is found that

$$
\begin{aligned}
E_{c}(k)= & E_{c}^{(0)}(k)+E_{c}^{(1)}(k)+\text { h.o.t., } \\
E_{c}^{(0)}(k)= & \frac{8 \pi N}{3 a} k^{-1} \exp \left(-\frac{2 D k^{2}}{3 a}\right) \\
& \times \sum_{n=1}^{\infty} \int_{0}^{\infty}\left|\hat{c}_{n}^{(0)}(\rho)\right|^{2} \rho d \rho, \\
E_{c}^{(1)}(k)= & \frac{16 \pi N}{3 a^{2 / 3}} k^{-5 / 3} \exp \left(-\frac{D k^{2}}{3 a}\right) \\
& \times \sum_{n=1}^{\infty} n^{2 / 3} \int_{0}^{\infty}|\Lambda(\rho)|^{2 / 3} \Re\left(\hat{c}_{n}^{(0)} P_{n}^{*}\right) d \rho, \\
P_{n}=i & \sum_{m=}^{\infty} \exp \left(-\frac{Q_{m n} k^{2}}{3 a}\right) \\
\times & \left.\frac{f_{m}}{m \Lambda^{2}} \frac{\partial \hat{c}_{n-m}^{(0)}}{\partial \rho}-\frac{(n-m)}{m^{2}} \frac{\partial}{\partial \rho}\left(\frac{f_{m}}{\Lambda^{2}}\right) \hat{c}_{n-m}^{(0)}\right), \\
Q_{m n}= & \frac{\nu m^{2}+D(n-m)^{2}}{n^{2}} .
\end{aligned}
$$

The scalar dissipation is

$$
\boldsymbol{\epsilon}_{c} \equiv \boldsymbol{\epsilon}_{c}^{(0)}+\boldsymbol{\epsilon}_{c}^{(1)}=2 D \int_{0}^{\infty} k^{2}\left(E_{c}^{(0)}(k)+E_{c}^{(1)}(k)\right) d k,
$$

from which we find that

$$
\begin{aligned}
\boldsymbol{\epsilon}_{c}^{(0)}= & 4 \pi N \sum_{n=1}^{\infty} \int_{0}^{\infty}\left|\hat{c}_{n}^{(0)}(\rho)\right|^{2} \rho d \rho, \\
\boldsymbol{\epsilon}_{c}^{(1)}= & \frac{16 \pi N \Gamma[2 / 3] D^{1 / 3}}{3^{1 / 3}} \sum_{n=1}^{\infty} n^{2} \\
& \times \int_{0}^{\infty}|\Lambda(\rho)|^{2 / 3} \mathfrak{R}\left(\hat{c}_{n}^{(0)} \mathcal{P}_{n}^{*}\right) d \rho,
\end{aligned}
$$

$$
\begin{aligned}
\mathcal{P}_{n}= & i \sum_{-\infty, m \neq 0}^{\infty} \frac{1}{\left((n-m)^{2}+n^{2}+m^{2} \mathrm{Sc}\right)^{2 / 3}} \\
& \times\left(\frac{f_{m}}{m \Lambda^{2}} \frac{\partial \hat{c}_{n-m}^{(0)}}{\partial \rho}-\frac{(n-m)}{m^{2}} \frac{\partial}{\partial \rho}\left(\frac{f_{m}}{\Lambda^{2}}\right) \hat{c}_{n-m}^{(0)}\right) .
\end{aligned}
$$

The dissipation added by the nonaxisymmetric spiral motions, $\epsilon_{c}^{(1)}$, scales as $D^{1 / 3}$ and is independent of the strain rate $a$. Therefore $\epsilon_{c}^{(1)} \rightarrow 0$ when $D \rightarrow 0$ at any fixed Sc. At large Reynolds number and $\mathrm{Sc} \geqslant 1, \epsilon_{c}=\epsilon_{c}^{(0)}$ approximately.

\section{A. The $k^{-1}$ component}

The component $E_{c}^{(0)}(k)$ shows the Batchelor ${ }^{15}$ form. It is produced by the winding of the initial scalar field by the axisymmetric vortex core, but surprisingly, is independent of the azimuthal velocity distribution in the core. $\epsilon_{c}^{(0)}$ is independent of both $D$ and the strain rate $a$. A short calculation shows that it is equal to the scalar variance $\overline{\left(c^{(0)}\right)^{2}}$ at $t=0$, as required. Combining (82) and (87) gives

$$
E_{c}^{(0)}(k)=\frac{2}{3} a^{-1} \epsilon_{c}^{(0)} k^{-1} \exp \left(-\frac{2 D k^{2}}{3 a}\right) .
$$

Equation (90) would be the same as Batchelors ${ }^{15}$ result if $3 a / 2$ was replaced by $-\gamma$, where $\gamma$ is the smallest principal rate of strain. Batchelor takes $\gamma=-0.5(\epsilon / \nu)^{1 / 2}$ based on some experimental results. Presently, for strain rates appropriate to the viscous-diffusive range, we take $a$ as equal to the average strain rate in one direction

$$
a=\left(\frac{\epsilon}{15 \nu}\right)^{1 / 2}
$$

for which $3 a / 2 \approx 0.387(\epsilon / \nu)^{1 / 2}$.

\section{B. The $\boldsymbol{k}^{-5 / 3}$ component}

The component (83) shows a $k^{-5 / 3}$ Corrsin-Obukov form but with coefficient which depends in a complicated way on the structures of both the scalar and the vorticity and also on $\nu$ and $D$ through the exponential factors in (84). This component of $E_{c}(k)$ arises from an interaction of the $O\left(\tau^{0}\right)$ and the $O\left(\tau^{-1}\right)$ terms in (76). We assume $a^{2}=O(\epsilon / \nu)$, and consider two cases (i) $\mathrm{Sc} \sim O(1), D=O(\nu) . E_{c}^{(1)}(k)$ is then cutoff by the exponential prefactor in (83) at wave numbers $k=O\left(\nu^{3} / \epsilon\right)^{-1 / 4}$, consistent with standard scaling arguments (see Tennekes and Lumley ${ }^{14}$ ). (ii) $\mathrm{Sc} \gg 1, D \ll \nu$. If it is assumed that the dominant term in the sum occurs near $n=1$, $m= \pm 1$ (this is true in the example discussed below), then the $E_{c}^{(1)}(k)$ spectrum will be cutoff by the corresponding terms in the $P_{n}$ sum, again at $k=O\left(\nu^{3} / \epsilon\right)^{-1 / 4}$. The $E_{c}^{(0)}(k)$ term has no exponential containing $\nu$ and will extend to the inverse Batchelor scale $k_{c}=O\left(D^{2} \nu / \epsilon\right)^{-1 / 4}$.

We can give an illustrative example of an initial scalar distribution which will show that a nonzero component of the $k^{-5 / 3}$ prefactor in (83) for the inertial-convective range, will in general require correlations between the structure of the scalar and the vorticity fields. Set $D=\nu=0$, and consider a scalar field described initially by 
$c(r, \theta, 0)$

$$
=c_{0} \exp \left(-\frac{\left(r \cos \theta-r_{0} \cos \beta\right)^{2}+\left(r \sin \theta-r_{0} \sin \beta\right)^{2}}{2 \sigma^{2}}\right) .
$$

This is a Gaussian scalar blob of width $2 \sigma$ centered at $r$ $=r_{0}, \theta=\beta$ in the normal plane. The Fourier coefficients are

$$
\begin{aligned}
& \hat{c}_{c}^{(0)}(r)=c_{0} \exp (-i n \beta) \mathcal{C}_{n}(r), \\
& \mathcal{C}_{n}(r)=c_{0} \exp \left(-\frac{r^{2}+r_{0}^{2}}{2 \sigma^{2}}\right) I_{n}\left(\frac{r_{0} r}{\sigma^{2}}\right),
\end{aligned}
$$

where $I_{n}(\cdots)$ is the $I$-Bessel function. We consider only the Fourier coefficients $n \neq 0$ and write the functions $f_{n}(r)$ in the Fourier coefficients for the vorticity, (20) as

$$
f_{n}(r)=\exp (-i n \gamma) \mathcal{F}_{n}(r),
$$

where the $\mathcal{F}_{n}(r)$ are real functions and $\gamma$ is a phase angle which fixes the orientation of the nonaxisymmetric component of the vorticity in the $(r-\theta)$ plane. A short calculation then shows that the factor $\hat{c}_{n}^{(0)} P_{n}^{*}$ inside the integrals of (83) can be written as

$$
\begin{aligned}
\hat{c}_{c}^{(0)} P_{n}^{*}= & -i c_{0}^{2} \mathcal{C}_{n}(\rho) \sum_{m=-\infty, \neq 0}^{\infty} e^{i m(\gamma-\beta)}\left(\frac{\mathcal{F}_{m}}{m \Lambda^{2}} \frac{\partial \mathcal{C}_{n-m}}{\partial \rho}\right. \\
& \left.-\frac{(n-m)}{m^{2}} \frac{\partial}{\partial \rho}\left(\frac{\mathcal{F}_{m}}{\Lambda^{2}}\right) \mathcal{C}_{n-m}\right) .
\end{aligned}
$$

If $r_{0}$ is fixed, and $\beta=\gamma \pm \pi$, (96) has zero real part and so $E_{c}^{(1)}(k)$ vanishes. Likewise if $\beta$ is fixed and (96) is averaged over $\gamma$ assumed uniformly distributed in $0 \leqslant \gamma \leqslant 2 \pi$, then $E_{c}^{(1)}(k)$ is again zero. This would correspond to all azimuthal positions of the scalar blob with respect to the nonaxisymmetric vorticity equally likely. A nonzero $E_{c}^{(1)}(k)$ would then require $\beta-\gamma$ correlations, which amounts to scalar-vorticity correlations. An example is $\gamma=\beta+\alpha$ with $\gamma, \beta$ both uniform on the unit circle. This will give a finite coefficient of $k^{-5 / 3}$ for almost all $\alpha$. It is easy to see that a general nonaxisymmetric initial scalar distribution must have Fourier coefficients that can be expressed like (93), with real functions $\mathcal{C}_{n}(r)$. Thus the above conclusions will hold for any arbitrary initial scalar.

\section{Estimate of the scalar spectrum}

A much simpler example, which can be carried further, has a specific correlation between the scalar and the vorticity. Assume that

$$
\omega_{3}(r, \theta, 0)=2 f_{0} g(\rho) \sin (2 \theta),
$$

where $f_{0}$ is a dimensional constant and $g(\rho)$ a structure function. This makes $f_{2}=-i f_{0} g, f_{-2}=i f_{0} g$ and all other $f_{n}=0$. Assume also that

$$
c(r, \theta, 0)=2 c_{0} g(\rho) \cos \theta,
$$

where $c_{0}$ is a dimensional constant and $g(\rho)$ is the same structure function. This makes $c_{1}=c_{0} g, c_{-1}=c_{0} g$ and all other $c_{n}=0$. In (83) only the $n=1$ term is nonzero, while in
$P_{1}$ from (84) only $m=2$ makes a contribution; therefore there is only one term in the double series. This results in

$$
\begin{aligned}
& E_{c}^{(0)}(k)=\frac{8 \pi N}{3 a} k^{-1} \exp \left(-\frac{2 D k^{2}}{3 a}\right) c_{0}^{2} A_{0} \\
& E_{c}^{(1)}(k)=\frac{16 \pi N}{3 a^{2 / 3}} k^{-5 / 3} \exp \left(-\frac{(4 \nu+2 D) k^{2}}{3 a}\right) f_{0} c_{0}^{2} B_{0} \\
& \epsilon_{c}^{(0)}=4 \pi N c_{0}^{2} A_{0} \\
& \epsilon_{c}^{(1)}=\frac{16 \pi N \Gamma[2 / 3] D^{1 / 3} f_{0} c_{0}^{2}}{3^{1 / 3}\left(2+4 S_{c}\right)^{2 / 3}} B_{0} \\
& A_{0}=\int_{0}^{\infty} g(\rho)^{2} \rho d \rho, \\
& B_{0}=\int_{0}^{\infty}|\Lambda(\rho)|^{2 / 3}\left(\frac{g^{2}}{2 \Lambda^{2}} \frac{\partial g}{\partial \rho}+\frac{g^{2}}{4} \frac{\partial}{\partial \rho} \frac{g}{\Lambda^{2}}\right) d \rho
\end{aligned}
$$

We evaluate the integrals $A_{0}$ and $B_{0}$ for a special case; $g(\rho)=1$ for $R / 2<\rho<R, \quad g(\rho)=0$ otherwise, and $\Lambda$ $=\Gamma / \pi \rho^{3}$ (a line vortex with circulation $\Gamma$ ). This gives

$$
\begin{aligned}
& A_{0}=\frac{3}{8} R^{2}, \\
& B_{0}=\frac{15}{128} \frac{\pi^{4 / 3} R^{4}}{\Gamma^{4 / 3}} .
\end{aligned}
$$

We further specialize these results by assuming $a$ $=(\epsilon / 15 \nu)^{1 / 2}$ and $f_{0}=\Gamma / R^{2}$. Then

$$
\begin{aligned}
E_{c}^{(0)}= & 2.58 \epsilon_{c}^{(0)} \nu^{1 / 2} \epsilon^{-1 / 2} k^{-1} \exp \left(-2.58 S_{c}^{-1}(k \eta)^{2}\right), \\
E_{c}^{(1)}= & \frac{4.71}{(\Gamma / \nu)^{1 / 3}} \epsilon_{c}^{(0)} \epsilon^{-1 / 3} k^{-5 / 3} \\
& \times \exp \left(-2.58\left(2+s_{c}^{-1}\right)(k \eta)^{2}\right), \\
\frac{\epsilon_{c}^{(1)}}{\epsilon_{c}^{(0)}}= & \frac{2.14}{(\Gamma / \nu)^{1 / 3}} \frac{1}{S_{c}^{1 / 3}\left(0.5+S_{c}\right)^{2 / 3}} .
\end{aligned}
$$

These results are independent of the constant $c_{0}$. If $\Gamma / \nu$ $=1000$, and $S_{c}$ is moderately large so that $\epsilon_{c} \simeq \epsilon_{c}^{(0)}$ then (108) has the Corrsin-Obukov form with a Corrsin-Obukov constant of 0.47 which comparable to the experimental value 0.58 found by Gibson and Schwarz. ${ }^{16}$ These results are plotted in Fig. 2 for $\Gamma / \nu=1000$ and two values of the Schmidt number, $S_{c}=7$ (heat in water), and $S_{C}=700$ (salt in water). The function plotted is

$$
\begin{aligned}
\frac{E_{c}(x) \epsilon^{3 / 4}}{\epsilon_{c}^{(0)} \nu^{5 / 4}}= & 2.58 x^{-1} \exp \left(-2.58 S_{c}^{-1} x^{2}\right) \\
& +0.471 x^{-5 / 3} \exp \left(-2.58\left(0.5+S_{c}^{-1}\right) x^{2}\right),
\end{aligned}
$$

where $x=k \eta$. It may be seen that, over the range of $k \eta$ plotted, the scalar spectrum does not exhibit a pure $-5 / 3$ 


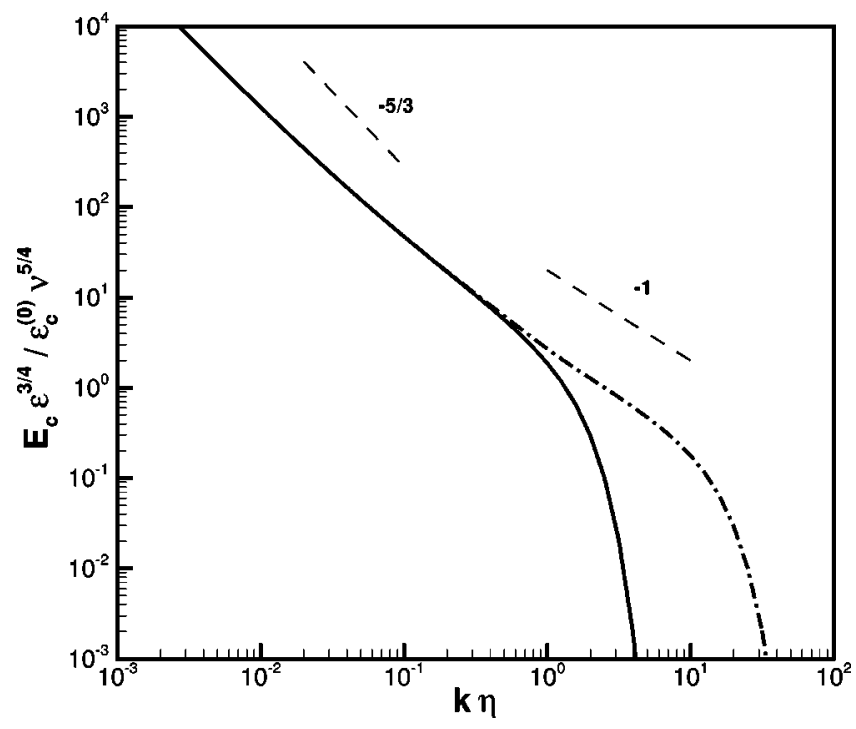

FIG. 2. Scalar spectrum, Eq. (110). $\Gamma / \nu=1000$. Solid, $\mathrm{Sc}=7$. Dasheddotted, $\mathrm{Sc}=700$.

range, owing to contamination by the $k^{-1}$ component. For $\mathrm{Sc}=700$, there is a short $k^{-1}$ range for $k \eta$ above which the $-5 / 3$ component has rolled off. The one-dimensional scalar spectrum can be calculated from

$$
G_{c}(k)=\int_{k}^{\infty} \frac{E_{c}(u)}{u} d u .
$$

Using (110) this gives

$$
\begin{aligned}
\frac{G_{c}(x) \epsilon^{3 / 4}}{\epsilon_{c}^{(0)} \nu^{5 / 4}}= & \frac{2.07}{\mathrm{Sc}^{1 / 2}} \Gamma\left[-\frac{1}{2}, 2.58 S_{c}^{-1} x^{2}\right] \\
& +\frac{0.519}{\left(0.5+S_{c}^{-1}\right)^{5 / 6}} \Gamma\left[-\frac{5}{6}, 2.58\left(0.5+S_{c}^{-1}\right) x^{2}\right],
\end{aligned}
$$

where $x=k_{1} \eta$ and

$$
\Gamma[\alpha, y]=\int_{y}^{\infty} t^{\alpha-1} e^{-t} d t
$$

is the incomplete gamma function. These results are shown in Fig. 3 compared to the data of Gibson and Schwartz. ${ }^{16}$ The agreement is satisfactory.

In this example, the correlation between scalar and vorticity is very specific. If we had used $\cos (2 \theta)$ instead of $\sin (2 \theta)$ in (97) we would have found $E_{c}^{(1)}$ equal zero. Our choice makes the following arrangement of the two structures. The initial axial vorticity is positive in the first and third quadrants and negative in the other two. The vorticity rolls up into spiraling vorticity layers of alternating sign. The scalar is positive in the right half plane and negative in the left. The scalar likewise is rolled up into layers of alternating sign. Therefore in each layer of positive or negative scalar are found two vorticity layers of opposite sign. The velocity field induced by the vortex layers is such as to produce maximum shearing velocity in the center of each scalar layer and

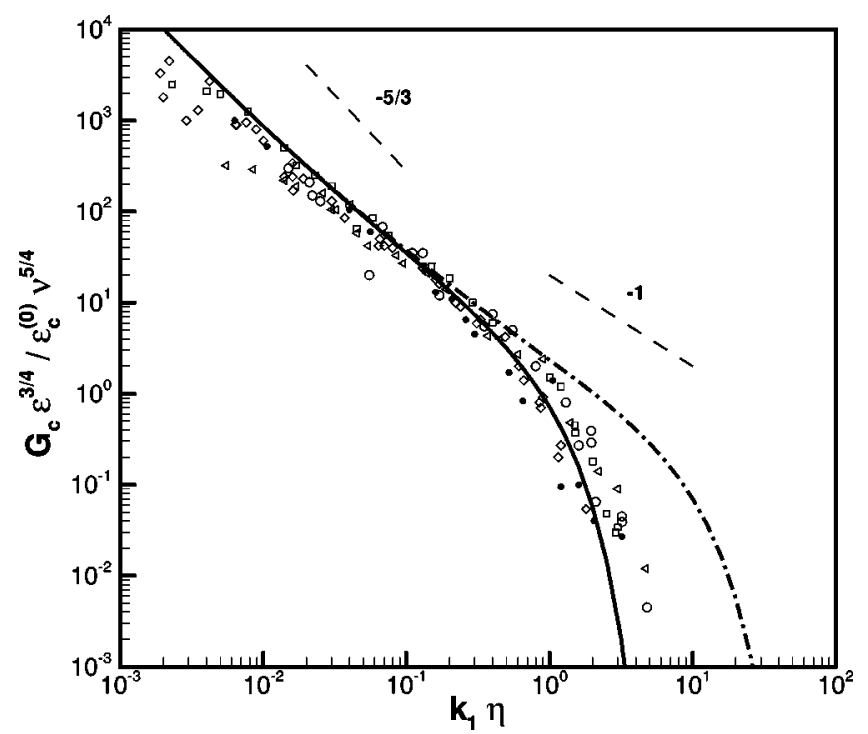

FIG. 3. One-dimensional scalar spectrum, Eq. (112). $\Gamma / \nu=1000$. Solid, $\mathrm{Sc}=7$. Dashed-dotted, $\mathrm{Sc}=700$. Symbols, data (Ref. 16).

velocity of the opposite sign at the edges where the scalar changes sign. This arrangement appears to produce maximum scalar-vorticity correlation.

\section{CONCLUDING REMARKS}

The main results of this paper are Eqs. (66) for the spectrum of the axial velocity, and Eqs. (66)-(85) giving the spectrum of a passive scalar mixed by the velocity field of the stretched spiral vortex. The axial velocity spectrum shows a $k^{-7 / 3}$ form. At large wave numbers this is subdominant in comparison to the $k^{-5 / 3}$ spectrum for the azimuthal velocity derived from the nonaxisymmetric component of the axial vorticity. Estimates of the mean square vorticity for the axial and the azimuthal components, which are proportional to the energy dissipation provided, indicates that these can be of equal order.

The spectrum of the passive scalar is the sum of two components. The first is a $k^{-1}$ spectrum produced by deformation of the initial scalar field; this is independent of the axial vorticity and contains a single parameter, the background rate of strain. When this strain rate is scaled in dissipation-range variables, Batchelor's spectral form for the convective-diffusive range is found. The second component is a $k^{-5 / 3}$ contribution arising from the interaction of the leading order perturbation term in the large-time asymptotic expansion of the scalar evolution, with the $O(1)$ term. This result depends on the detailed spiral structure of the nonaxisymmetric part of the axial vorticity. Correlations of the scalar with the nonaxisymmetric part of the axial vorticity are found necessary in order to generate a nonzero coefficient of the $k^{-5 / 3}$ part of the scalar spectrum.

It is notable that the model produces a scalar spectrum that is the sum of two power laws. This may explain why it has proved difficult to find experimentally, a pure $-5 / 3$ scalar spectrum in the inertial-convective range. Finally, it is of interest that the stretched spiral vortex is able to unify several 
classical results in turbulence including the Kolmogorov velocity spectrum, and both the Obukov-Corrsin and Batchelor scalar spectra.

\section{ACKNOWLEDGMENT}

D.I.P. was supported in part by the National Science Foundation under Grant No. CTS-9978551.

${ }^{1}$ T. S. Lundgren, "Strained spiral vortex model for turbulent fine structure," Phys. Fluids 25, 2193 (1982).

${ }^{2}$ T. S. Lundgren, "The concentration spectrum of the product of a fast biomolecular reaction," Chem. Eng. Sci. 40, 1641 (1985).

${ }^{3}$ D. I. Pullin and P. G. Saffman, "On the Lundgren-Townsend model of turbulent fine scales," Phys. Fluids A 5, 126 (1993).

${ }^{4}$ T. S. Lundgren, "A small-scale turbulence model," Phys. Fluids A 5, 1472 (1993).

${ }^{5}$ J. D. Buntine, D. I. Pullin, and P. G. Saffman, "On the spectrum of a stretched spiral vortex," Phys. Fluids 6, 3010 (1994).

${ }^{6}$ D. I. Pullin, "Pressure spectra for vortex models of homogeneous turbulence," Phys. Fluids 7, 849 (1995).

${ }^{7}$ D. I. Pullin and P. G. Saffman, "Vortex dynamics in turbulence," Annu. Rev. Fluid Mech. 30, 31 (1998).

${ }^{8} \mathrm{D}$. Segel, "The higher moments in the Lundgren model conform with Kolmogorov scaling," Phys. Fluids 7, 3072 (1995).

${ }^{9}$ S. Kida, "Vortical structure in turbulence," presented at ICTAM 2000, Chicago, September 2000.

${ }^{10}$ C. F. Pearson and F. H. Abernathy, "Evolution of the flow field associated with a streamwise diffusing vortex," J. Fluid Mech. 146, 271 (1984).
${ }^{11} \mathrm{D}$. W. Moore, "The interaction of a diffusing line vortex and an aligned shear flow," Proc. R. Soc. London, Ser. A 399, 367 (1985).

${ }^{12}$ M. Tanaka, G. Kawahara, S. Kida, and S. Yanase, "Wrap, tilt and stretch of vorticity lines around a strong straight vortex tube in a simple shear flow," J. Fluid Mech. 353, 115 (1997).

${ }^{13}$ A. S. Fokas, J. D. Gibbon, and C. R. Doering, "Dynamically stretched vortices as solutions of the 3d Navier-Stokes equations," Physica D 132, 497 (1999).

${ }^{14} \mathrm{H}$. Tennekes and J. L. Lumley, A First Course in Turbulence (MIT Press, Cambridge, MA, 1974).

${ }^{15} \mathrm{G}$. K. Batchelor, "Small-scale variation of convected quantities like temperature in turbulent fluid. Part I. General discussion and the case of small conductivity," J. Fluid Mech. 5, 113 (1959).

${ }^{16}$ C. H. Gibson and W. H. Schwarz, "The universal equilibrium spectra of turbulent velocity and scalar fields," J. Fluid Mech. 16, 365 (1963).

${ }^{17}$ W. M. Vogel, H. L. Grant, B. A. Hughes, and A. Moilliet, "The spectrum of temperature fluctuations in turbulent flow," J. Fluid Mech. 34, 423 (1968).

${ }^{18}$ G. Brethouwer and F. T. M. Nieuwstadt, "Mixing of weakly and strongly diffusive passive scalars in isotropic turbulence," in Direct and Large Eddy Simulation III, edited by N. D. Landham P. R. Noke, and L. Kleiser (Kluwer Academic, New York, 1999), pp. 311-322.

${ }^{19}$ A. Misra and D. I. Pullin, "A vortex-based subgrid stress model for largeeddy simulation," Phys. Fluids 9, 2443 (1997).

${ }^{20} \mathrm{~K}$. Bajer, "Flux expulsion by a point vortex," Eur. J. Mech. B/Fluids 17, 653 (1998).

${ }^{21} \mathrm{P}$. B. Rhines and W. R. Young, "How rapidly is a passive scalar mixed within closed streamlines?" J. Fluid Mech. 133, 113 (1983).

${ }^{22}$ P. Flohr and J. C. Vassilicos, "Accelerated scalar dissipation in a vortex," J. Fluid Mech. 348, 295 (1997). 